\title{
Depth-of-Field Enhancement in Integral Imaging by Selective Depth-Deconvolution
}

\author{
Hector Navarro, Genaro Saavedra, Manuel Martínez-Corral, Mårten Sjöström, Member, IEEE, and \\ Roger Olsson, Member, IEEE
}

\begin{abstract}
One of the major drawbacks of the integral imaging technique is its limited depth of field. Such limitation is imposed by the numerical aperture of the microlenses. In this paper, we propose a method to extend the depth of field of integral imaging systems in the reconstruction stage. The method is based on the combination of deconvolution tools and depth filtering of each elemental image using disparity map information. We demonstrate our proposal presenting digital reconstructions of a 3-D scene focused at different depths with extended depth of field.
\end{abstract}

Index Terms-Deconvolution, disparity map, depth-of-field (DOF), integral imaging (InI).

\section{INTRODUCTION}

I $\mathrm{N}$ THIS PAPER we present a new method to extend the depth-of-field (DOF) of integral imaging (InI) systems in the reconstruction stage. Deconvolution tools have been previously used to extend the DOF of InI systems, but these tools are only effective in situations where all the elements in the $3 \mathrm{D}$ scene are affected by the same blur in the capture stage. The novelty of this work is that our method can be used in situations where the blur strongly depends on the axial position of the elements in the $3 \mathrm{D}$ scene.

From time immemorial, man has sought to reflect the world around as faithfully as possible. Along history have appeared different techniques to reproduce images, being these increasingly realistic. First displays that humans used to exhibit images were the walls and ceilings of caves around 40,000 years ago. Greeks and Romans may have understood linear perspective and Filippo Brunelleschi rediscovered it sometime close to 1420 . Detailed instructions about linear perspective were published by Leon Battista Alberti in his painting manual "De Pictura" [1]. Leonardo da Vinci highlighted the need to use both eyes to perceive images in three dimensions in his work

Manuscript received January 25, 2013; revised April 22, 2013, September 06, 2013, October 23, 2013; accepted November 06, 2013. Date of publication November 14, 2013; date of current version February 11, 2014. This work was supported in part by the Plan Nacional I+D+I(Grant DPI2012-32994), Ministerio de Economía y Competitividad (Spain) and by the Generalitat Valenciana(Grant PROMETEO2009-077). The work of H. Navarro was supported by the Generalitat Valenciana (VALi $+d$ predoctoral contract).

H. Navarro, G. Saavedra, and M. Martinez-Corral are with the Departmen of Optics, University of Valencia, E-46100 Burjassot, Spain (e-mail: hector. navarro@uv.es; genaro.saavedra@uv.es; manuel.martinez@uv.es).

M. Sjöström and R. Olsson are with the Department of Information Technology and Media, Mid Sweden University, SE-851 70 Sundsvall, Sweden (e-mail: marten.sjostrom@miun.se; roger.olsson@miun.se).

Color versions of one or more of the figures are available online at http:// ieeexplore.ieee.org.

Digital Object Identifier 10.1109/JDT.2013.2291110
"Tratato della Pintura" [2]. An important milestone was the invention of the monochrome photographic camera by Joseph Nicéphore Niépce. In the XIX century, Wheatstone designed the first system that took into account the influence of binocular vision [3]. Realism increased with the invention of the first method to reproduce color in photography [4]. This method was invented by Gabriel Lippmann and led him to win the Nobel Prize in Physics in 1908. It was precisely in the same year when Lippmann published his paper "Épreuves réversibles donnant la sensation $d u$ relief" [5], where he proposed a novel 3D imaging technique that laid the groundwork for what today is known as InI. This method works with incoherent illumination and hence can capture and display true color 3D images [6]. InI provides stereo parallax as well as full and continuous motion parallax, allowing multiple viewing positions for several viewers. The Lippmann idea was placing a photographic plate behind an array of small magnifying glasses. These lenses are placed next to each other generating what is known as fly's-eye lens array. Each lenslet images a different perspective of a $3 \mathrm{D}$ scene over the photographic plate. After developing the plate, the resulting image is placed behind the same fly's-eye lens array. An observer placed in front of this system sees the original 3D image in its original position and of the same size.

A limited DOF is a serious problem for InI because to reconstruct clear 3D images it is essential to capture sharp 2D elemental images (EI). Objects belonging to the 3D scene located in axial positions out of the DOF will appear blurred in the EI, and hence, will also be blurred in the reconstructed image. The easiest way to increase the depth of field would be reducing the numerical aperture of the microlenses. However, this improvement is accompanied by a proportional reduction of the light-collection efficiency and a deterioration of the lateral resolution. Some alternative methods have been proposed to increase the DOF of an InI system. These methods are based on the synthesis of real and virtual image fields [7], the use of lenses with non-uniform focal length and sizes [8], [9], the use of multilayered display devices [10] and the modulation of the amplitude transmittance of the microlenses together with deconvolution tools [11], [12]. Our proposal to extend the DOF consists in reversing the out-of-focus blur by a selective depth deconvolution. First we obtain the disparity map of every EI to know the axial position of each object. The axial range spanning the scene is divided into intervals and then each EI is filtered by selecting the pixels that are associated with a given interval. It is possible to define an effective point spread function (PSF) in good approximation over each interval of distances. Only the pixels belonging to a certain interval are deconvolved with the PSF calculated for the center of the interval. The final image is 
the sum of the pixels of every interval after being filtered and deconvolved. This procedure has been applied to a set of EIs captured with the synthetic aperture InI (SAII) method proposed in [13]. Computational reconstructions focused at different depths show the extension in the DOF.

The paper is organized as follows. In Section II, we present a diffractive analysis of the capture setup. Section III explains the methodology applied to extend the DOF and Section IV shows reconstructions focused at different depths with enhanced DOF. Conclusions are presented in Section V.

\section{Diffractive ANALysis OF SAII}

To discuss the method we start by describing the capture stage from the point of view of diffraction theory. When using an array of microlenses, each lenslet can be considered as a thin lens and the calculation of its impulse response is straightforward [14]. As stated in the introduction, instead of using a microlens array we have used a SAII method in which a digital camera is mechanically translated over a plane perpendicular to the optical axis. For the calculation of the impulse response of a camera it must be taken into account that a camera lens, even the simplest, is an assembly of lenses and a multi-leaf diaphragm and all these elements are embedded inside a cover. Furthermore, some of the lenses can move inside the cover, so that the diffractive analysis becomes slightly complicated. This may seem a disadvantage, but SAII has multiple advantages such as the possibility of changing the focal length, the aperture of the diaphragm, the ability for focusing at different depths and the correction of the optical distortions. In order to reduce the complexity of the diffractive analysis of the pick-up stage with SAII, we propose a model that greatly simplifies the calculations and provides accurate results. From experimental parameters such as the diameter of the entrance pupil of the camera lens, its distance to the in-focus plane and the magnification factor between the in-focus plane and the sensor, the impulse response of the camera lens can be easily obtained. The most relevant elements of a camera from the point of view of diffraction are shown in Fig. 1. The entrance pupil is the image of the diaphragm through lenses that precede it. All rays passing through the entrance pupil will traverse the entire optical system so that it can be considered as the window through which light enters the objective. Let us suppose that the camera shown in Fig. 1 is focusing on a reference plane located at a distance $\alpha$ from the entrance pupil of the camera lens. Because the diaphragm is a circular stop, the entrance pupil has also circular shape.

Consider the light wave scattered at an arbitrary point $\left(x_{0}, y_{0}, z_{0}\right)$ of the surface of a 3D object (see Fig. 2). For simplicity we assume quasi-monochromatic illumination with mean wavelength $\lambda$. Spatial coordinates are denoted $(x, y)$ and $z$ for directions transverse and parallel to the system main optical axis. The amplitude distribution of the spherical wave emitted by that point in the plane of the entrance pupil can be written as

$$
\begin{aligned}
U_{E}^{\lambda}(x, y ; z)= & -\frac{1}{z+\alpha} e^{-i k(z+\alpha)} \\
& \cdot \exp \left\{-i \frac{k}{2(z+\alpha)}\left(x^{2}+y^{2}\right)\right\} p_{E}(x, y)
\end{aligned}
$$

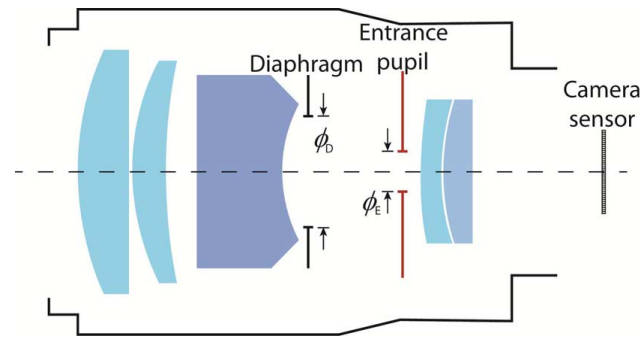

Fig. 1. Camera lens scheme showing the diafragm and the image of its opening throught lenses in front of it.

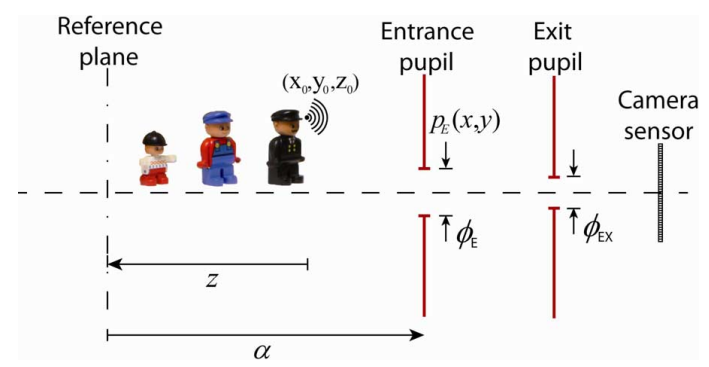

Fig. 2. Scheme of the capture setup. Object points out of the reference plane produce blurred images in the sensor.

where $k=2 \pi / \lambda$ is the wave number. In this equation we have multiplied the impinging wave-front by the amplitude transmittance of the entrance pupil, $p_{E}(x, y)$. In order to simplify resulting equations we will use a mathematical artifice consisting on a virtual propagation from the plane of the entrance pupil to the reference plane. The virtual amplitude distribution in the reference plane is given by

$$
\begin{aligned}
U_{R}^{\lambda}(x, y ; z)= & \frac{e^{i k z}}{(z+\alpha) \alpha} \exp \left\{-i \frac{k}{2 \alpha}\left(x^{2}+y^{2}\right)\right\} \\
& \cdot \int_{-\infty}^{+\infty} p_{E}\left(x_{0}, y_{0}\right) \exp \left\{-i \frac{k}{2} \frac{z}{(z+\alpha) \alpha}\left(x_{0}^{2}+y_{0}^{2}\right)\right\} \\
& \cdot \exp \left\{i \frac{2 \pi}{\lambda \alpha}\left(x x_{0}+y y_{0}\right)\right\} d x_{0} d y_{0}
\end{aligned}
$$

Since the reference plane and the camera sensor are conjugated through the camera lens, the amplitude distribution over the sensor is a scaled version of the amplitude distribution at the reference plane

$$
U_{S}^{\lambda}(x, y ; z)=\frac{1}{M_{0}} U_{R}^{\lambda}\left(\frac{x}{M_{0}}, \frac{y}{M_{0}} ; z\right) .
$$

The scaling factor $M_{0}$ is given by the lateral magnification between these two planes. Introducing (2) into (3), we straightforwardly find that

$$
\begin{aligned}
U_{S}^{\lambda}(x, y ; z) & =\frac{e^{i k z}}{M_{0}(z+\alpha) \alpha} \exp \left\{-i \frac{k}{2 M_{0}^{2} \alpha}\left(x^{2}+y^{2}\right)\right\} \\
& \cdot \int_{-\infty}^{+\infty} p_{E}\left(x_{0}, y_{0}\right) \exp \left\{-i \frac{k}{2} \frac{z}{(z+\alpha) \alpha}\left(x_{0}^{2}+y_{0}^{2}\right)\right\} \\
& \cdot \exp \left\{i \frac{2 \pi}{\lambda M_{0} \alpha}\left(x x_{0}+y y_{0}\right)\right\} d x_{0} d y_{0}
\end{aligned}
$$


If a local reference system that moves with the camera is used, impulse response has radial symmetry around the optical axis of the camera lens. Therefore using cylindrical coordinates is best suited to write these equations. Accordingly, (4) can be rewritten as

$$
\begin{aligned}
U_{S}^{\lambda}(r ; z)= & \frac{2 \pi e^{i k z}}{M_{0}(z+\alpha) \alpha} \exp \left\{-i \frac{k}{2 M_{0}^{2} \alpha} r^{2}\right\} \\
& \cdot \int_{0}^{\phi_{E} / 2} p_{E}(\rho) \exp \left\{-i \frac{\pi}{\lambda} \frac{z}{(z+\alpha) \alpha} \rho^{2}\right\} \\
& \cdot J_{0}\left(\frac{2 \pi}{\lambda \alpha M_{0}} r \rho\right) \rho d \rho
\end{aligned}
$$

where $\phi_{\mathrm{E}}$ is the diameter of the entrance pupil of the camera lens and $r$ is the radial coordinate over the sensor plane. The response generated in the sensor of the camera is proportional to the intensity of the incident light. Therefore, the intensity impulse response can be obtained as the squared modulus of the function in (5).

$$
H_{S}^{\lambda}(r ; z)=\left|U_{S}^{\lambda}(r ; z)\right|^{2}
$$

Function $H_{S}^{\lambda}(r ; z)$ has a strong dependence on the axial position of the corresponding surface points. Consequently, the impulse response is different at different depths. Therefore, the 3D PSF cannot be rigorously defined. However, the impulse response can be regarded as the sum of the impulse responses generated by a continuum of point sources axially distributed. This fact can be written as

$$
H_{S}^{\lambda}(r, z)=\int H_{S}^{\lambda}(r, \varepsilon) \delta(z-\varepsilon) d \varepsilon
$$

From this interpretation, it is possible to define the intensity PSF for each depth with respect to the reference plane, which is precisely $H_{S}^{\lambda}(r, \varepsilon)$. Given a plane at a distance $z=\varepsilon$ from the reference plane, $\Pi_{\varepsilon}(r, \theta)$, its image $I$ over the sensor can be expressed as the $2 \mathrm{D}$ convolution of a scaled version of the intensity distribution scattered at that plane, $\Pi_{\epsilon}\left(r / M_{\varepsilon}, \theta\right)$, and the function $H_{S}^{\lambda}(r, \varepsilon)$

$$
I_{S}^{\lambda, \varepsilon}=\frac{1}{M_{\varepsilon}^{2}} \Pi_{\varepsilon}\left(\frac{r}{M_{\varepsilon}}, \theta\right) \otimes_{r, \theta} H_{S}^{\lambda}(r, \varepsilon)
$$

The scaling factor $M_{\varepsilon}$ comes from the lateral magnification of the camera lens and depends on the distance $\varepsilon$ from the plane of interest to the reference plane.

We will extend now our analysis to a volumetric object. Suppose a photo camera whose sensor is conjugated through the camera lens with some plane cutting the object in two parts. The intensity distribution of incoherent light scattered by the object surface can be represented by the real and positive function $O(r, \theta, z)$. This function can be expressed as

$$
O(r, \theta, z)=\int O(r, \theta, \varepsilon) \delta(z-\varepsilon) d \varepsilon
$$

which can be interpreted as if the object has been sliced in infinitesimal width sheets. From (8), the image of one of these sheets over the sensor can be written as

$$
I_{S}^{\lambda, O_{\varepsilon}}(r, \theta)=\frac{1}{M_{\varepsilon}^{2}} \delta(z-\varepsilon) O\left(\frac{r}{M_{\varepsilon}}, \theta, z\right) \otimes_{r, \theta} H_{S}^{\lambda}(r, \varepsilon) .
$$

The image of the object over the sensor can be considered as the sum of the images of each slice of the object over the sensor. The intensity distribution corresponding to the image of the object onto the sensor plane is given by

$$
\begin{aligned}
& I_{S}^{\lambda, O}(r, \theta) \\
& \quad=\frac{1}{M_{\varepsilon}^{2}} \int \delta(z-\varepsilon) O\left(\frac{r}{M_{\varepsilon}}, \theta, z\right) \otimes_{r, \theta} H_{S}^{\lambda}(\rho, \varepsilon) d \varepsilon .
\end{aligned}
$$

On the sensor plane we obtain a superposition of the image of the in-focus plane and the blurred images of the rest of sections that constitute the $3 \mathrm{D}$ scene.

It should be noted that the previous diffractive analysis can only be applied if the optical aberrations are not significant within the depth of field in which the experiment is performed. This is not a problem when working with a SAII system, because a high performance camera lens is used to capture each EI. When using a microlens array with spherical lenses, some shift-variant aberrations are present, which affect differently the PSF at different lateral positions over the EI plane. A solution for this problem consists of using a microlens array with aspherical microlenses. In such case, the main aberrations might be quite well corrected and the analysis here presented can be considered as a good approximation of the actual behavior of the optical system.

\section{Deconvolution AND DePth FILTERING}

Let us consider a 2D slice of a 3D scene, which is perpendicular to the optical axis. As stated in (10), the image of this slice over the sensor can be expressed as the $2 \mathrm{D}$ convolution of the intensity distribution at that plane with the intensity PSF associated with that depth. By knowing the PSF for that depth, it is possible to reverse out-of-focus blur caused by the optical system. There is a wide variety of deconvolution methods, but in order to minimize the impact of the photon noise in the restored image we will use Richardson-Lucy deconvolution [15], [16]. This method cannot be applied to simultaneously recover a sharp version of objects located at different depths if those objects are affected by different blur. However, if it is possible to identify which depth in the scene is associated with each pixel of the image, pixels associated with the surfaces located at a given depth can be selected, and the corresponding deconvolution for that depth can be applied. In order to perform this process, we will use the method proposed by Shin et al. in [17]. An integral image may be considered as a set of stereo pairs, and accordingly well developed stereo vision algorithms can be used to get the disparity map of each EI. From the camera parameters and taking into account that the disparity is inversely proportional to the depth, is straightforward obtaining the depth information of the different objects composing the scene. The 


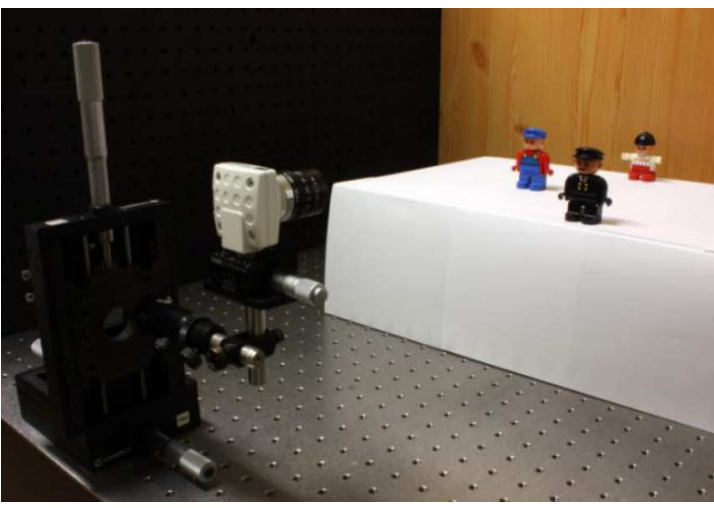

Fig. 3. Experimental setup for the acquisition of the EIs of a 3D scene with SAII method.

disparity map resolution scales linearly with the EI resolution and therefore this technique is best suited for capturing systems such as multi-camera arrays or SAII where the EIs are captured with high resolution. The increase in the EI resolution also increases the storage and bandwidth requirements. A solution to this problem was recently proposed by Cho et al. in [18], where Compressive Sensing is used to dramatically reduce the amount of data that needs to be collected to form the EIs.

The heart of any stereo vision system is stereo matching, the goal of which is to establish correspondence between two points arising from the same element in the scene. Stereo matching is usually complicated by several factors such as lack of texture, occlusion, discontinuity and noise. Basic block matching chooses the optimal disparity for each pixel based on its own cost function alone, but this technique creates a noisy disparity image [19]. To improve these results we have used the optimization method proposed by Veksler [20], in which the disparity estimation at one pixel depends on the disparity estimation at the adjacent pixels. The maximum number of distances which can be distinguished is given by the number of bits used in the disparity map. For example, if working with 8 bit disparity map, it is possible to distinguish 256 distances in the range covering the scene. But for our purpose, it is usually not necessary to use all these intervals. As the complexity of the surfaces of the objects composing the $3 \mathrm{D}$ scene increases, we must increase the number of axial intervals in which we divide the scene. Accordingly, it is useful to approximate the intensity distribution of the 3D objects using constant depth segment as

$$
O(x, y, z)=O\left(x, y, z_{i}\right), \quad z_{i} \leq z<z_{i+1}, \quad i=1, \ldots, N
$$

where $N$ is the number of intervals and the object is confined between $z_{0}$ and $z_{N+1}$. Only the pixels belonging to a certain interval are deconvolved in the EI with the effective PSF calculated for the center of that interval.

\section{SAII RECONSTRUCTIONS WITH IMPROVED DOF}

To validate the proposed method we performed a hybrid experiment in which the EIs were captured experimentally and the reconstruction was simulated computationally. In Fig. 3, we show the experimental setup for the pickup stage. As can be seen in the picture, the scene was composed by a wooden back-
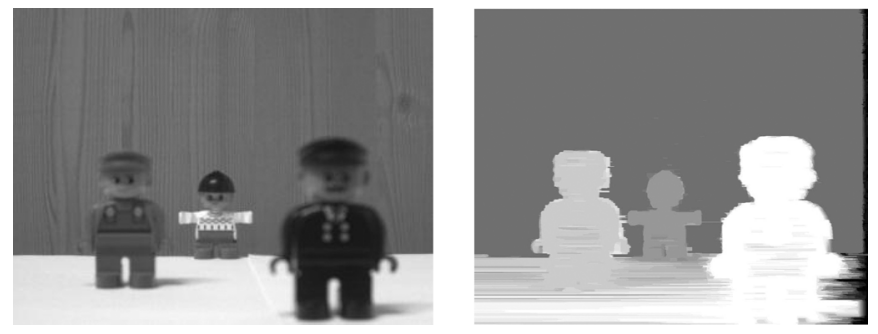

Fig. 4. EI with shallow DOF captured with SAII method (Left). Disparity map for the EI on the left (Right).

ground and three toy figures located at different distances from the camera. A digital camera was mechanically translated in a rectangular grid. It was accurately calibrated so that the optical axis was perpendicular to the plane defined by the grid in which the camera is moved. A set of $11 \times 11$ EIs of $2560 \times 1920$ pixels each was taken with a camera lens of fixed focal length $f=12 \mathrm{~mm}$. The sensor of the camera was conjugated with the wooden surface in the background which was located at a distance $\alpha=745.4 \mathrm{~mm}$ from the entrance pupil of the camera lens.

The $\mathrm{f} /$ number of the camera lens was chosen to $f \#=2.8$, and the lateral magnification for the in-focus plane was $M_{0}=$ -0.019 . Finally, the entrance pupil of the camera lens was measured with the help of a microscope, obtaining a diameter $\emptyset_{E}=$ $4.7 \mathrm{~mm}$. In the left image of Fig. 4 we show one of the captured EIs for these settings. As stated in the previous section an integral image can be regarded as a collection of stereo pairs. According to this we can apply Veksler method to the various stereo pairs composing the integral image in order to get the disparity map of each EI. As an example, in the right image of Fig. 4 we present the disparity map of the EI on the left.

The depth information of the objects in the $3 \mathrm{D}$ scene is obtained from the disparity map and the parameters of the experimental setup. The scene is mainly composed by three objects located at different depths plus a background and therefore, according to (12), it can be divided in four intervals. As shown in the diffractive analysis of the capture stage the intensity PSF associated with a particular interval can be calculated theoretically. The distance $z$ of the center of the various intervals to the in-focus plane is obtained from the depth information. For each EI, pixels associated with a given interval are deconvolved with its corresponding intensity PSF obtained from (6). The result of this process can be seen in Fig. 5 .

The final EI with extended DOF is obtained as the sum of the pixels of the various intervals after being filtered and deconvolved. Fig. 6 shows the EI of Fig. 4 with extended DOF.

In Fig. 6, there are two features that lead to a poor visual aspect. Abrupt transitions are due to the border of the areas selected in the disparity map for each axial interval and the ringing is caused by the calculated PSFs oscillating features, which causing ripples in the image at rapidly changing intensities. The image quality could be addressed by using a deconvolution kernel with less oscillating tap values as long as it retains the correct geometrical support. These features are attenuated when performing computational reconstructions of the $3 \mathrm{D}$ scene focused at different depths.

The back-projection technique described in [21] is used to reconstruct the scene focused at different depths. The collec- 

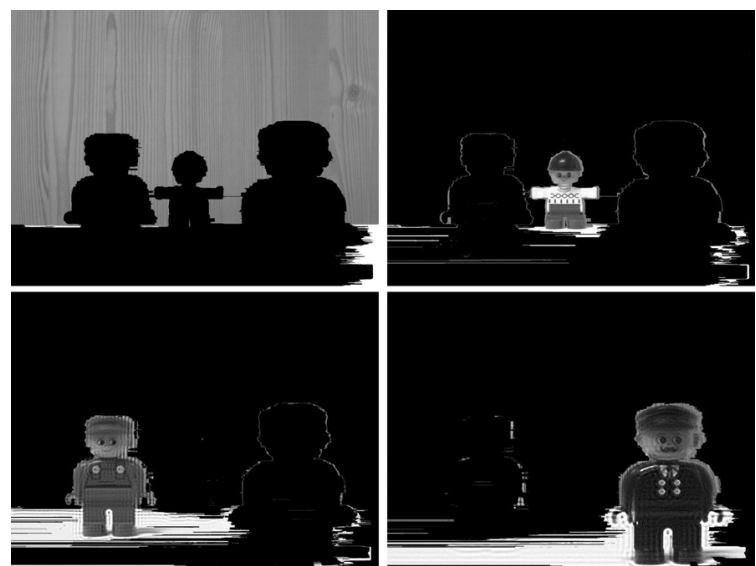

Fig. 5. Filtering and deconvolution of the EI in Fig. 4 for each axial interval.

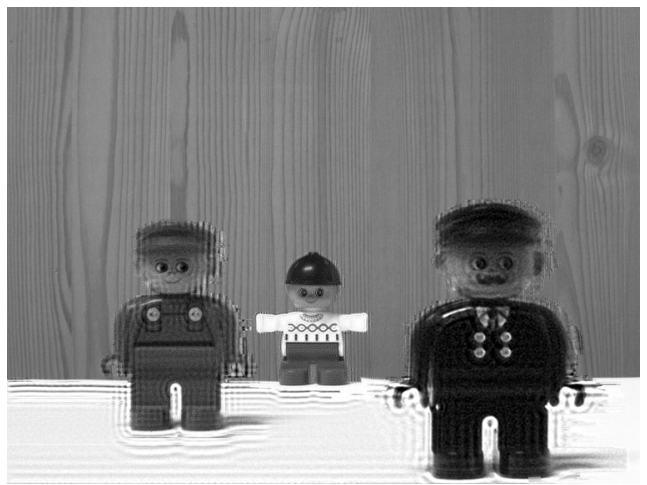

Fig. 6. EI of Fig. 4 with extended DOF using selective depth-deconvolution method.

tion of all back-projected EIs is superimposed computationally to achieve the intensity distribution on the reconstruction plane [22]. In the reconstruction stage, ringing effects and abrupt transitions are averaged and smoothed, improving the visual appearance of the reconstructed images. In Fig. 7, we show a collection of reconstructions of the captured scene for the depths where the toy figures where located. A comparison of the reconstructions obtained with and without applying our method is presented.

Comparing the EI in the left side of Fig. 4 with the reconstructions in the left column of Fig. 7, it can be seen that parts of the scene that appear blurred in the EI appear also blurred in the reconstructions, producing deterioration in the lateral resolution of the reconstructed images. To illustrate the improvement in the lateral resolution, an enlarged version of the areas enclosed by the lines in Fig. 7 is presented in Fig. 8. From top to bottom of the left column of Fig. 8, it can be seen how the blur increases as the distance of the toy figures to the in focus plane becomes larger. In the right column we show the result of applying selective depth-deconvolution to each EI and the computational reconstruct of the scene at the same depths than in the left column. By comparing these reconstructions, one can observe that in the right column it is possible to resolve details that in the left column are impossible to distinguish. These results prove the ability of our method to extend the DOF in the reconstructions stage.

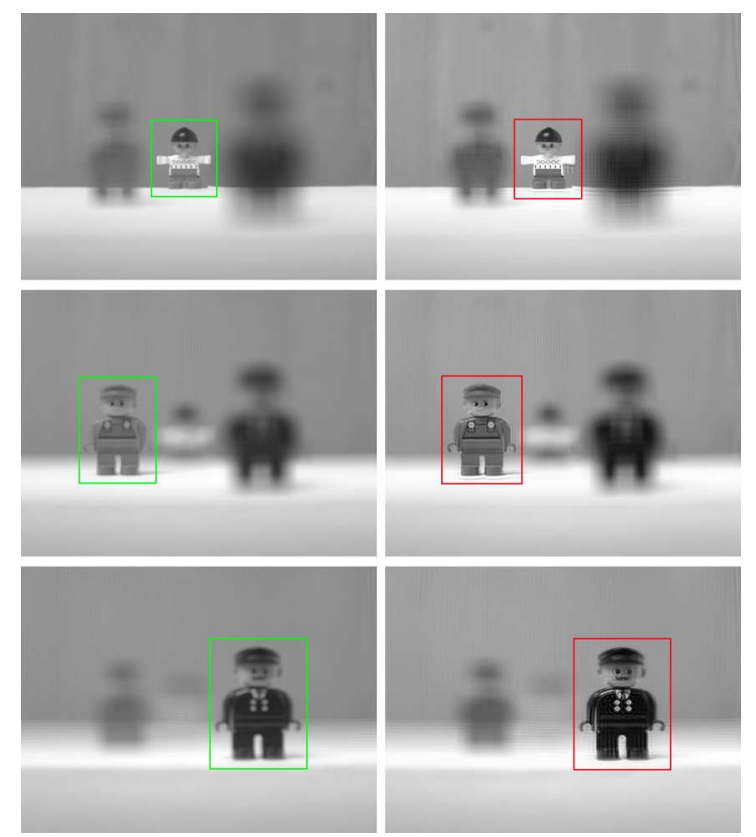

Fig. 7. Computational reconstructions at different depths from the captured EIs (left column). Computational reconstructions at different depths after applying the proposed method to each EI (right column).
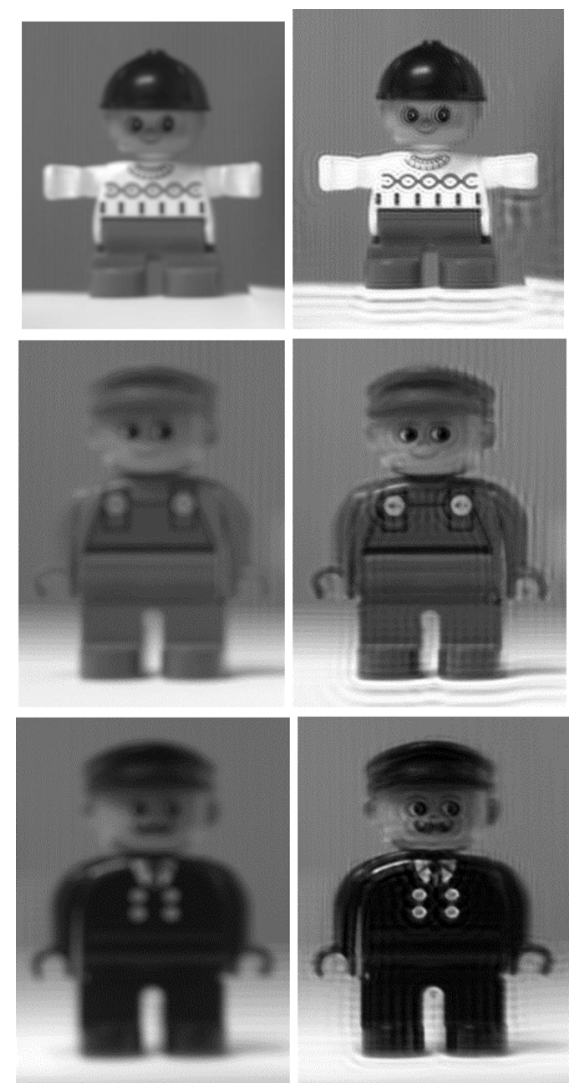

Fig. 8. Enlarged version of the areas enclosed by the lines in the left column of Fig. 7 (left column). Enlarged version of the areas enclosed by the lines in the right column of Fig. 7 (right column).

\section{CONCLUSION}

We present a method to extend the DOF of an InI system in the reconstruction stage. A set of EIs with shallow DOF was 
captured experimentally. The method is based in the combination of deconvolution tools and stereo vision algorithms. This technique is best suited for capturing systems such as multicamera arrays or SAII. Using a back-projection algorithm, we have simulated reconstructions of a 3D scene focused at different depths. We have recovered details of the objects reconstructed at different depths that without applying the proposed technique cannot be resolved. In future work we intend to quantify the extension in the DOF by analyzing the frequency content of the reconstructed images.

\section{REFERENCES}

[1] L. B. Alberti, De Pictura 1436.

[2] L. da Vinci, Trattato della Pintura [preceded by a life of the author by C. Amoretti], L. da Vinci and R. du Fresne, Eds. Milan, Italy: Società Tipografica de' Classici Italiani, 1804.

[3] C. Wheatstone, "Contributions to the physiology of vision," Philos. Trans. Roy. Soc. London, vol. 4, pp. 76-77, 1837.

[4] G. Lippmann, "La photographie des couleurs," in Comptes Rendues des Scéances de l'Acad. Sciences, Paris, France, 1891, vol. 112, pp. 274-275.

[5] G. Lippmann, "Epreuves reversibles donnant la sensation du relief," $J$. Phys, vol. 7, pp. 821-825, 1908.

[6] , B. Javidi and F. Okano, Eds., Three-Dimensional Television, Video and Display Technologies. Berlin, Germany: Springer-Verlag, 2002.

[7] J.-S. Jang, F. Jin, and B. Javidi, "Three-dimensional integral imaging with large depth of focus by use of real and virtual image fields," Opt. Lett., vol. 28, pp. 1421-1423, 2003.

[8] B. Lee, S.-W. Min, S. Jung, and J.-H. Park, "Computer-generated dynamic three-dimensional displays using integral photography adopting Fresnel lenses," in Proc. SPIE Algorithms Syst. for Opt. Inf. Process. $V$, 2001, vol. 4471, pp. 9-17.

[9] J.-S. Jang and B. Javidi, "Large depth of focus time-multiplexed three-dimensional integral imaging by use of lenslets with non-uniform length and aperture sizes," Opt. Lett., vol. 28, pp. 1924-1926, 2003.

[10] Y. Kim, J.-H. Park, H. Choi, J. Kim, S.-W. Cho, and B. Lee, "Depthenhanced three-dimensional integral imaging by use of multilayered display devices," Appl. Opt., vol. 45, pp. 4334-4343, 2006.

[11] M. Martínez-Corral, B. Javidi, R. Martínez-Cuenca, and G. Saavedra, "Integral imaging with improved depth of field by use of amplitudemodulated microlens array," Appl. Opt., vol. 43, pp. 5806-5813, 2004.

[12] R. Martínez-Cuenca, G. Saavedra, M. Martínez-Corral, and B. Javidi, "Extended depth-of-field 3-D display and visualization by combination of amplitude-modulated microlenses and deconvolution tools," J. Display Technol., vol. 1, no. 2, pp. 321-327, Dec. 2005.

[13] J.-S. Jang and B. Javidi, "Three-dimensional synthetic aperture integral imaging," Opt. Lett., vol. 27, pp. 1144-1146, 2002.

[14] J. W. Goodman, Introduction to Fourier Optics, 3rd ed. Greenwood Village, CO, USA: Roberts, 2005, pp. 109-111.

[15] W. H. Richardson, "Bayesian-based iterative method of image restoration," J. Opt. Soc. Amer., vol. 62, pp. 55-59, 1972.

[16] L. B. Lucy, "An iterative technique for the rectification of observed images," Astronom. J., vol. 79, pp. 745-754, 1974.

[17] D.-H. Shin, B.-G. Lee, and J.-J. Lee, "Occlusion removal method of partially occluded 3D object using sub-image block matching in computational integral imaging," Opt. Exp., vol. 16, pp. 16294-16304, 2008.

[18] M. Cho, A. Mahalanobis, and B. Javidi, "3D passive integral imaging using compressive sensing,” Opt. Express, vol. 20, pp. 26624-26635, 2012.

[19] E. Trucco and A. Verri, Introductory Techniques for 3-D Computer Vision. Upper Saddle River, NJ, USA: Prentice Hall, 1998.

[20] O. Veksler, "Stereo correspondence by dynamic programming on a tree," Proc. IEEE Conf. Comput. Vis. Pattern Recogn., vol. 2, pp. 384-390, 2005.

[21] S.-H. Hong, J.-S. Jang, and B. Javidi, "Three-dimensional volumetric object reconstruction using computational integral imaging," Opt. Express, vol. 12, pp. 483-491, 2004.
[22] H. Navarro, G. Saavedra, A. Molina, M. Martinez-Corral, R. MartinezCuenca, and B. Javidi, "Optical slicing of large scenes by synthetic aperture integral imaging," in Proc. SPIE, 2010, vol. 7690, p. 7690-0M.

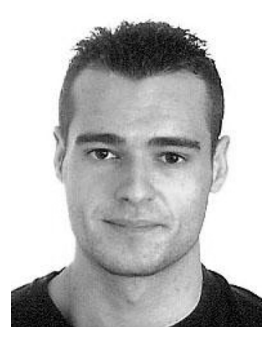

Hector Navarro received the B.Sc. and M.Sc. degrees in physics from the University of Valencia, Spain, in 2008 and 2009, respectively. Since 2007 he has been with the 3D Imaging and Display Laboratory at the Optics Department of the University of Valencia. H. Navarro is member of SPIE since 2010. His research interests include focusing properties of light and 3D imaging acquisition and display. He has published 11 articles in major journals and authored 18 communications in prestigious Physics Conferences.

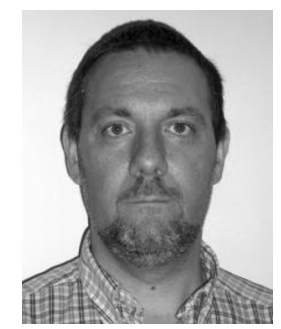

Genaro Saavedra received the B.Sc. and Ph.D. degrees in physics from Universitat de València, Spain, in 1990 and 1996, respectively. His Ph.D. work was honored with the Ph.D. Extraordinary Award. He is currently an Associate Professor with this University. Since 1999, he has been working with the "3D Display and Imaging Laboratory", at the Optics Department. His current research interests are optical diffraction, integral imaging, 3D high-resolution optical microscopy and phase-space representation of scalar optical fields. He has published on these topics about 50 technical articles in major journals and 3 chapters in scientific books. He has published over 50 conference proceedings, including 10 invited presentations.

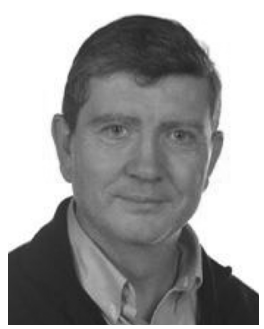

Manuel Martinez-Corral was born in Spain in 1962. He received the M.Sc. and Ph.D. degrees in physics from the University of Valencia in 1988 and 1993, respectively. In 1993, the University of Valencia honored him with the Ph.D. Extraordinary Award. He is currently Full Professor of Optics at the University of Valencia, where he is with the "3D Imaging and Display Laboratory". His research interest includes scalar and vector properties of tightly focused light fields, resolution procedures in $3 \mathrm{D}$ scanning microscopy, and $3 \mathrm{D}$ imaging and display technologies. He has supervised on these topics six Ph.D. theses, two of them honored with the Ph.D. Extraordinary Award, published over 70 technical articles in major journals, and pronounced over 20 invited and 5 keynote presentations in international meetings. He has been member of the Scientific Committee in over 15 international meetings, and was the president of the Organizing Committee of the international conference Focus on Microscopy 2007. He is co-chair of the Three-Dimensional Imaging, Visualization, and Display Conference within the SPIE meeting in Defense, Security, and Sensing (Baltimore). He is Topical Editor of the IEEE/OSA JOURNAL OF DISPLAY TECHNOLOGY. In 2010, he was named Fellow of the SPIE.

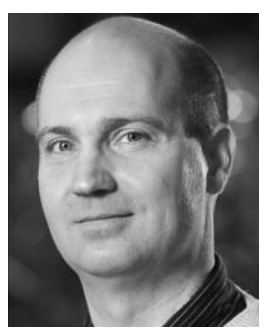

Mårten Sjöström (S'95-A'01-M'03) received the M.Sc. degree in electrical engineering and applied physics from Linköping University, Sweden, in 1992, the Licentiate of Technology degree in signal processing from KTH, Stockholm, Sweden, in 1998, and the Ph.D. degree in modeling of nonlinear systems from EPFL, Lausanne, Switzerland, in 2001. He worked as an Electrical Engineer at ABB, Sweden, from 1993-1994, was a fellow at CERN from 1994-1996, and a Ph.D.-student at EPFL, Lausanne, Switzerland during 1997-2001. In 20011, he joined the Department of Information Technology and Media, Mid Sweden University as a Senior Lecturer. During 2002-2006, he was appointed Head and Assistant Head of Division, respectively, and since 2008, he is Associate Professor. His current research interests are within system modelling and identification, as well as 2D and 3D image and video processing. 


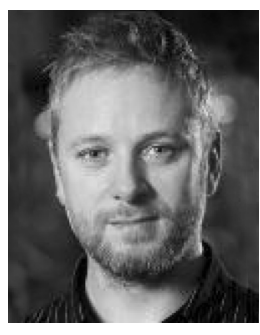

Roger Olsson (GSM'06-M'08) received the M.Sc. in electrical engineering and $\mathrm{Ph} . \mathrm{D}$. in telecommunications from Mid Sweden University (MIUN) in 1998 and 2010 respectively. During 1998-2000 he worked as a research engineer at Limt Technology $\mathrm{AB}$ with compression and MPEG-2 TS stream splicing in digital TV distribution systems. He joined the Department of Information Technology and Media at MIUN in 2000, working as a lecturer in signal processing and telecommunications until 2004. His Ph.D. studies were pursued within the

field of integral imaging and he is a founding member of the Realistic 3D research group at MIUN. His research interests include plenoptic image capture, modeling, processing, compression, and presentation; Time-Of-Flight depth data processing; and depth-image-based rendering. He is a member of the IEEE since 2006. 